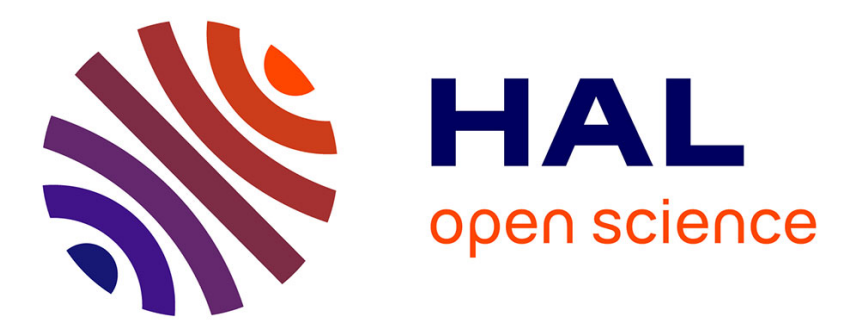

\title{
Regularized friction and continuation: Comparison with Coulomb's law
}

Pierre Vigué, Christophe Vergez, Sami Karkar, Bruno Cochelin

\section{To cite this version:}

Pierre Vigué, Christophe Vergez, Sami Karkar, Bruno Cochelin. Regularized friction and continuation: Comparison with Coulomb's law. Journal of Sound and Vibration, 2017, 389, pp.350-363. 10.1016/j.jsv.2016.11.002 . hal-01350658v2

\section{HAL Id: hal-01350658 \\ https://hal.science/hal-01350658v2}

Submitted on 9 Dec 2016

HAL is a multi-disciplinary open access archive for the deposit and dissemination of scientific research documents, whether they are published or not. The documents may come from teaching and research institutions in France or abroad, or from public or private research centers.
L'archive ouverte pluridisciplinaire HAL, est destinée au dépôt et à la diffusion de documents scientifiques de niveau recherche, publiés ou non, émanant des établissements d'enseignement et de recherche français ou étrangers, des laboratoires publics ou privés.

\section{(2)(1) $\$$}

Distributed under a Creative Commons Attribution - NonCommercial - ShareAlikel 4.0 


\title{
Regularized friction and continuation: Comparison with Coulomb's law
}

\author{
Pierre Vigué ${ }^{a}$, Christophe $\operatorname{Vergez}^{a}$, Sami Karkar ${ }^{b}$, Bruno Cochelin $^{a}$ \\ a : Aix Marseille Univ, CNRS, Centrale Marseille, LMA, Marseille, France \\ b : Ecole Centrale de Lyon, 36 avenue Guy de Collongue, 69134 Ecully Cedex, France \\ \{vigue, vergez\}@lma.cnrs-mrs.fr, sami.karkar@ec-lyon.fr, bruno.cochelin@centrale-marseille.fr
}

\begin{abstract}
Periodic solutions of systems with friction are difficult to investigate because of the non-smooth nature of friction laws. This paper examines periodic solutions and most notably stick-slip, on a simple one-degreeof-freedom system (mass, spring, damper, belt), with Coulomb's friction law, and with a regularized friction law (i.e. the friction coefficient becomes a function of relative speed, with a stiffness parameter). With Coulomb's law, the stick-slip solution is constructed step by step, which gives a usable existence condition. With the regularized law, the Asymptotic Numerical Method and the Harmonic Balance Method provide bifurcation diagrams with respect to the belt speed or normal force, and for several values of the regularization parameter. Formulations from the Coulomb case give the means of a comparison between regularized solutions and a standard reference. With an appropriate definition, regularized stick-slip motion exists, its amplitude increases with respect to the belt speed and its pulsation decreases with respect to the normal force.
\end{abstract}

\section{Introduction}

In scientific and engineering research, friction is a topic that dates back to da Vinci [1], and it is still a major issue in many systems. Brakes can create noise, such as the extensively studied brake squeal [2]. Friction plays a crucial role in mechanical joints [3]. The role of friction in acoustics has been reviewed in [4], with a wide range of examples, from the wineglass rubbed on its rim to the insects producing stridulatory sounds.

For the bowed string, the seminal works of Helmholtz (1877) and Raman (1918) describe several regimes, respectively Helmholtz's corner, and Raman's higher types. Several works have since attempted to describe the multitude of possible regimes. In addition to experimental observations with various artificial bowing apparatus (for example, a rosined perspex rod [5]), the main theoretical results [6] are established through graphic constructions (for instance, Friedlander's rule), numerical time simulation (time integration, digital waveguides...), or modal analysis [7].

However, to the authors' knowledge, there is no systematic numerical investigation of periodic solutions of the bowed string, which might be due to the non-smooth nature of friction laws. Yet as a highly nonlinear system, the bowed string may exhibit several solutions for the same set of bowing parameters, depending on initial conditions. For example, a string lightly touched by a finger at its middle emits a sound known as flageolet tone or harmonic sound that is the second register. Using appropriate bowing parameters, musicians can maintain this second register solution in the same bow stroke when the finger is raised, although these bowing parameters are compatible with a normal, first register sound. The authors' ultimate aim is the continuation of periodic solutions of a bowed string, showing the evolution of solutions with respect to a given parameter, along with their stability. A string toy model, based on a truncated modal projection retaining only two modes, was studied with the regularized law presented here [8].

The numerical framework presented in [9] will be used hereafter. It operates on first-order, parametric differential system $X^{\prime}=F(X, \lambda)$. A discretization method, namely, the Harmonic Balance Method or the Orthogonal Collocation at Gauss points, transforms this differential system into a polynomial one (also called algebraic system). Then, a continuation method, the Asymptotic Numerical Method (ANM), is used to study the evolution of periodic solutions. It relies on truncated power series expansion, provided that in the parametric differential system $X^{\prime}=F(X, \lambda), F$ is an analytic function, and thus, so is the solution branch. In its implementation, the ANM operates with quadratic nonlinearities, with an extension to usual functions 


\begin{tabular}{|l|l|l|}
\hline Symbol & Signification & Numerical value (if applicable) \\
\hline$\lambda$ & Continuation parameter & $\cdot$ \\
$V_{b}$ & Belt speed & $0.2 \mathrm{~m} . \mathrm{s}^{-1}\left(\right.$ if $\left.\lambda=F_{N}\right)$ \\
$F_{N}$ & Normal force & $5 \mathrm{~N}\left(\right.$ if $\left.\lambda=V_{b}\right)$ \\
$\zeta_{1}$ & Damping & $1.3096 \times 10^{-3}$ \\
$\omega_{1}$ & Natural pulsation & $1.2316 \times 10^{3} \mathrm{rad} . \mathrm{s}^{-1}$ \\
$M_{1}$ & Modal mass & $6.42 \times 10^{-3} \mathrm{~kg}$ \\
$\mu_{s}$ & Static friction coefficient & 0.4 \\
$\mu_{d}$ & Dynamic friction coefficient & 0.2 \\
$\varepsilon$ & Modulus smoothing parameter & $10^{-4}$ \\
$\alpha$ & Regularization constant & $\alpha=\sqrt{\mu_{s}\left(\mu_{s}-\mu_{d}\right)} \simeq 0.283$ \\
$n$ & Regularization parameter & $\cdot$ \\
\hline
\end{tabular}

[10], but keeping in mind that only smooth nonlinearities are admissible. That is why a regularized friction law is proposed that will be reviewed in this paper. It is not designed to fit experimental data, but rather, to present some similarities to Coulomb's two-parameters law (recalled in Eq. (3)). This regularized law engenders periodic solutions that can be compared with Coulomb's solution on a string toy model. Unlike Pennestri et al. [11], who compare through time integration many models for a given set of parameters values, the present study aims at presenting the global behaviour of one model family through continuation, with different values of the regularization parameter.

As a first step before a realistic string model, the present paper examines the periodic solutions of a massspring-damper resting on a conveyor belt. The model is presented is Section 2. This mass-spring-damper-belt device, also known as the Rayleigh string model, corresponds to the projection on the first mode of the string equation.

Explicit formulations of the periodic solution for this system with Coulomb's law are well-known in the undamped case. In the damped case however, there is a lack of such formulations, and instead of using ad hoc numerical integration techniques (for example, $[12,13]$ ), the authors describe explicitly the stick-slip solution, with Coulomb's law and damping, in Section 3. It is then sufficient to solve numerically only one equation (the cycle condition), and in return, it provides insight into the existence domain of this solution. The regularized law is given in Section 4, with a brief discussion about the regularization. In Section 5 we present the continuation of periodic solutions with the regularized law. The continuation is carried out with respect to the belt speed (Section 5.2), then to the normal force (Section 5.3). Periodic solutions obtained with the regularized law are compared with Coulomb's stick-slip solution.

\section{Model}

The model studied in this paper is a mass-spring-damper-belt system. The mass rests on a conveyor belt moving at velocity $V_{b}$ (Figure 1), and the contact between the mass and the belt follows a friction law $\mu$. This law is either :

- Coulomb's friction law, with distinct static and dynamic friction parameters $\mu_{s}, \mu_{d}$ in Section 3.

- a regularized (smooth) friction law $\mu_{n}$, presented and discussed in Section 4, used in Section 5. $n$ is the regularization parameter of the smooth friction law. For small values of $n$ the system is weakly nonlinear; for greater values of $n$ it becomes stiff.

The mass-spring-damper-belt system is a useful toy model of a bowed string. If $y$ denotes the horizontal transverse displacement at the bowing point $x=x_{b}$ of a string fixed at both ends, a projection of the equation of motion on the first bending mode leads to a mass-spring-damper equation. Namely, if $V_{r}$ denotes the relative velocity between the mass and the belt, $V_{b}$ the belt velocity, $F_{N}$ the normal force, $M_{1}$ the modal string mass, $\zeta_{1}$ the damping, $\omega_{1}$ the natural pulsation,

$$
y^{\prime \prime}+2 \zeta_{1} \omega_{1} y^{\prime}+\omega_{1}^{2} y=\frac{F_{N}}{M_{1}} \mu\left(V_{r}\right)
$$




$$
\text { where } V_{r}=y^{\prime}-V_{b}
$$

We underline that the normal force $F_{N}$ is not to be confused with gravity here : like in a musical context, the force applied by the bow is a parameter that can vary independently from the string mass. Moreover, gravity is negligible in comparison with the normal force.

a)

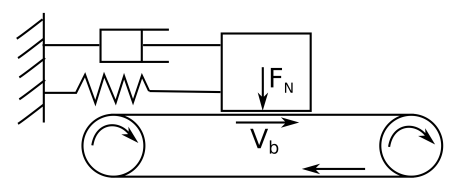

b)
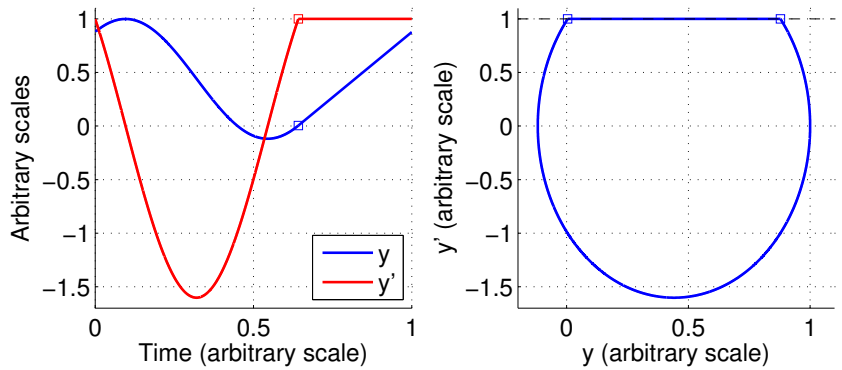

Figure 1: a) Mass-spring-damper system with a conveyor belt. b) One period of stick-slip motion associated with Coulomb's law $\left(V_{b}=0.1 \mathrm{~m} . \mathrm{s}^{-1} ;\right.$ arbitrary scales), squares indicate the limits of sticking.

\section{Periodic stick-slip solution using Coulomb's friction law}

\subsection{Coulomb's law}

In this section we study the periodic stick-slip solution of the mass-spring-damper-belt system with Coulomb's friction law, defining the friction coefficient as :

$$
\mu\left(V_{r}\right)= \begin{cases}-\mu_{d} \operatorname{sign}\left(V_{r}\right) & \text { if } V_{r} \neq 0 \text { (slip) } \\ \mu_{0} \text { with }\left|\mu_{0}\right| \leqslant \mu_{s}, & \text { if } V_{r}=0, \text { (stick) }\end{cases}
$$

with $\mu_{s}$ and $\mu_{d}$ respectively the static and dynamic friction coefficients, and $\mu_{d}<\mu_{s}$. An example of stick-slip periodic motion is drawn in Figure 1. This periodic motion can be described as follows. While the friction coefficient is smaller that $\mu_{s}$, the mass sticks to the belt $\left(V_{r}=0\right)$. When the friction coefficient reaches its maximum $\mu_{s}$, the restoring force of the stretched spring pulls back the mass that slips $\left(V_{r}<0, \mu=\mu_{d}\right)$. Then the direction of the mass changes, the compressed spring pushes the mass, and if the velocity of the mass reaches the belt velocity, the mass sticks again to the belt.

We define $F_{1}=\frac{F_{N}}{M_{1}}, \eta_{1}=\zeta_{1} \omega_{1}$, so that Eq. (1) becomes

$$
y^{\prime \prime}+2 \eta_{1} y^{\prime}+\omega_{1}^{2} y=F_{1} \mu\left(V_{r}\right)
$$

If the solution is slipping-only, $\forall t, V_{r}<0$, so the equation becomes

$$
y^{\prime \prime}+2 \eta_{1} y^{\prime}+\omega_{1}^{2} y=-F_{1} \mu_{d} \operatorname{sign}\left(V_{r}\right)=F_{1} \mu_{d}
$$

The right-hand side is constant, so this equation has one fixed-point solution, $y=\frac{F_{1} \mu_{d}}{\omega_{1}^{2}}$, and other solutions are damped oscillations, not periodic solutions. We determine the periodic stick-slip solution in three steps : stating the initial problem and its slipping solution ; determining whether slipping stops ; if it does, closing the cycle with a sticking phase. These three steps are detailed in the three subsections below. 


\subsection{Slipping}

We suppose that at $t=0$ the spring is stretched enough to end the sticking interval. This means $\mu\left(V_{r}\right)$ reaches at $t=0^{-}$its maximum $\mu_{s}$, and the slipping interval starts. Eq. (4) is to be solved with $\mu\left(V_{r}\right)=\mu_{s}$ on an interval $\left[0, t_{1}\right]$, where $t_{1}$, the instant when slipping stops, will be found later, and with initial conditions :

$$
\begin{aligned}
y(0) & =\omega_{1}^{-2}\left(F_{1} \mu_{s}-2 \eta_{1} V_{b}\right) \\
y^{\prime}(0) & =V_{b}
\end{aligned}
$$

The damped pulsation is noted $\omega_{A}:=\omega_{1} \sqrt{1-\zeta_{1}^{2}}$, there exists $A>0, \varphi \in[-\pi, \pi[$ so that

$$
y(t)=A e^{-\eta_{1} t} \cos \left(\omega_{A} t+\varphi\right)+\frac{F_{1} \mu_{d}}{\omega_{1}^{2}}
$$

and initial conditions give

$$
\begin{aligned}
& A \cos (\varphi)=y(0)-\frac{F_{1} \mu_{d}}{\omega_{1}^{2}} \\
& A \sin (\varphi)=-\omega_{A}^{-1}\left(y^{\prime}(0)+\eta_{1}\left(y(0)-\frac{F_{1} \mu_{d}}{\omega_{1}^{2}}\right)\right)
\end{aligned}
$$

so $A^{2}$ and $\tan (\varphi)$ are known. Then it can be shown that $\left.\varphi \in\right]-\frac{\pi}{2}, 0[$, and the unique couple $(A, \varphi) \in$ $\left(\mathbb{R}_{+}^{*},\right]-\frac{\pi}{2}, 0[)$ is now entirely determined by Eq. (8a), (8b).

\subsection{Does slipping stop?}

Now that the slipping interval has started, there is a dilemma : either there exists $t_{1}>0$ when slipping stops, or there is no such $t_{1}>0$.

The first case happens if $V_{r}$, which has become strictly negative during slipping, reaches 0 once more (cycle condition). It is also the first non-negative time $t$ where $y$ as given by Eq. (7) satisfies $y^{\prime}\left(t_{1}\right)=y^{\prime}(0)$. Then a stick-slip regime exists.

We recall that this resolution is simple in the undamped case ; in this case $t_{1}=\frac{\pi-2 \varphi}{\omega_{1}}$, stick-slip solution exists for all belt speeds ; and periodic solutions without sticking interval exist. These pure slipping solutions are located inside the stick-slip cycle in the phase diagram (see for example [14]). The value of $t_{1}$ in the undamped case can be used as a starting approximate value for a numerical solver in the damped case.

In the second case, there is no such $t_{1}>0$, meaning the damped oscillation of $y$ satisfies :

$$
\forall t>0, \quad y^{\prime}(t)<y^{\prime}(0)
$$

The system stays in a damped oscillation and there is no periodic solution. In the phase diagram (Figure 1, right), the left end of the horizontal segment (sticking interval) will not be reached. Inequation (9) is now transformed into a condition checked only at a specific time $t_{Y}$, instead of all times $t$.

$y^{\prime}$ can be rewritten as

$$
\begin{aligned}
y^{\prime}(t) & =-A \omega_{1} e^{-\eta_{1} t} \cos \left(\omega_{A} t+\varphi-\psi\right) \\
\text { with } \quad \psi & =\arccos \left(\eta_{1} \omega_{1}^{-1}\right)
\end{aligned}
$$

Then

$$
\text { No stick-slip } \Leftrightarrow(9) \Leftrightarrow \forall t>0, \quad y^{\prime}(t)=-A \omega_{1} e^{-\eta_{1} t} \cos \left(\omega_{A} t+\varphi-\psi\right)<y^{\prime}(0)=V_{b}
$$

Since the left-hand side is a damped oscillation, its first local maximum is its global maximum, and it occurs at $t=t_{Y}$ : 


$$
t_{Y}=\frac{2 \psi-\varphi+\frac{\pi}{2}}{\omega_{A}}
$$

From this we conclude :

$$
\text { No stick-slip } \Leftrightarrow A \exp \left(-\eta_{1} \frac{2 \psi-\varphi+\frac{\pi}{2}}{\omega_{A}}\right)<\frac{V_{b}}{\omega_{A}}
$$

The inequation (13) can be tested easily, and numerical results highlight that for a fixed normal force, a maximal belt speed $V_{\max }$ exists, beyond which stick-slip does not exist. Conversely, for a fixed belt speed, a minimal normal force $F_{\min }$ exists, below which there is no stick-slip. This does not provide an explicit expression of $V_{\max }$ or $F_{\min }$ in function of the other system parameters ${ }^{1}$, since $A$ and $\varphi$ depend on $V_{b}$ and $F_{N}$.

\subsection{Sticking}

a)

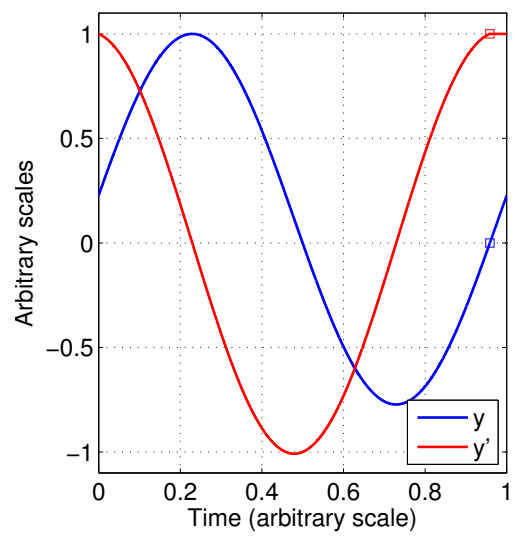

b)

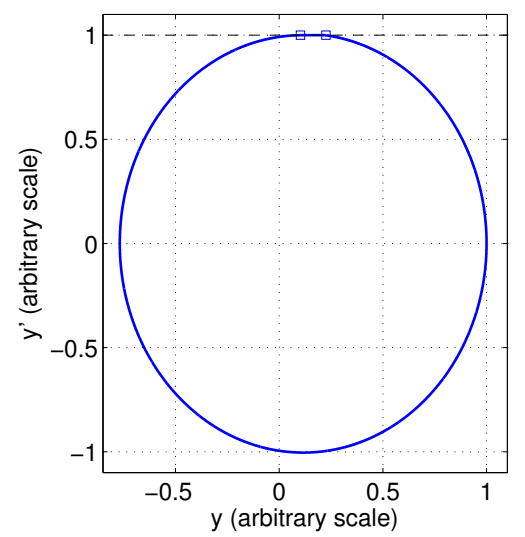

c)

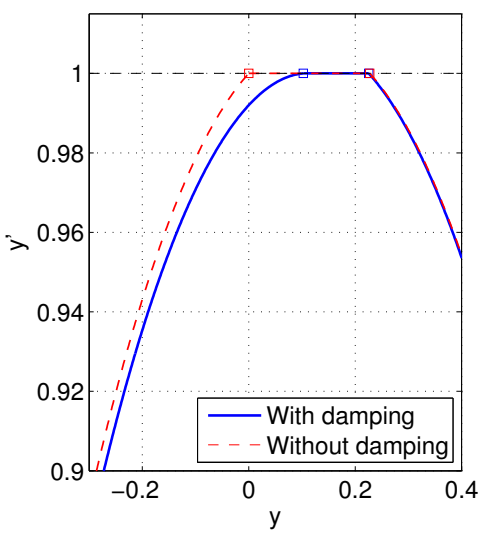

Figure 2: Stick-slip periodic solution of the system with Coulomb's friction law, with $V_{b}$ close to the maximal belt speed $V_{m a x}$. a) One period of $y$ (blue) and its derivative $y^{\prime}$ (red) (arbitrary scales), squares indicate $t=t_{1}$ (when sticking starts). b) Phase diagram $\left(y, y^{\prime}\right)$; dashed black line indicates adherence $\left(y^{\prime}=V_{b}\right)$, squares show the limits of sticking. c) Zoom on the sticking phase, comparison with the undamped case.

We conclude the study of the periodic case, when $t_{1}$ exists. Sticking starts at $t=t_{1}$, and stops at $t_{2}$, thus $T=t_{2}$ is the period of the cycle. For all $t \in\left[t_{1}, t_{2}\right], V_{r}(t)=0$, i.e. $y^{\prime}(t)=y^{\prime}(0): y$ is affine, increasing from its initial value $y\left(t_{1}\right)$ to its final value $y\left(t_{2}\right)=y(0)$. We calculate $A$ and $\varphi$ (Eq. (8)); $t_{1}$ is found with a numerical solver using the value in the undamped case as an initial guess ; now constants in Eq. (7) are known and so is $y\left(t_{1}\right)$, from which we conclude :

$$
t_{2}=t_{1}+\frac{y(0)-y\left(t_{1}\right)}{y^{\prime}(0)}
$$

Stick-slip solution $y$ over one period, its derivative $y^{\prime}$, and the phase diagram $\left(y, y^{\prime}\right)$ are plotted in Figure 2 for a fixed choice of parameters, and $V_{b}$ is close to the maximal belt speed $V_{\max }$. Two features can be underlined :

- since the belt speed is high, the sticking interval is then reduced to a short fraction of the period, unlike in Figure 1.

\footnotetext{
${ }^{1}$ We consider this is the most advanced, exact resolution to describe whether stick-slip exists or not. Wensrich [15] also points out the difficulty to obtain an explicit boundary between the two domains.
} 
- in the phase diagram, the damped solution has an almost horizontal tangent at the end of the slipping interval, unlike the undamped solution.

We can even compute the bifurcation diagram, for instance over a belt speed interval $\left[V_{1} ; V_{2}\right]$ (or, similarly a normal force interval) discretized in $k+1$ values :

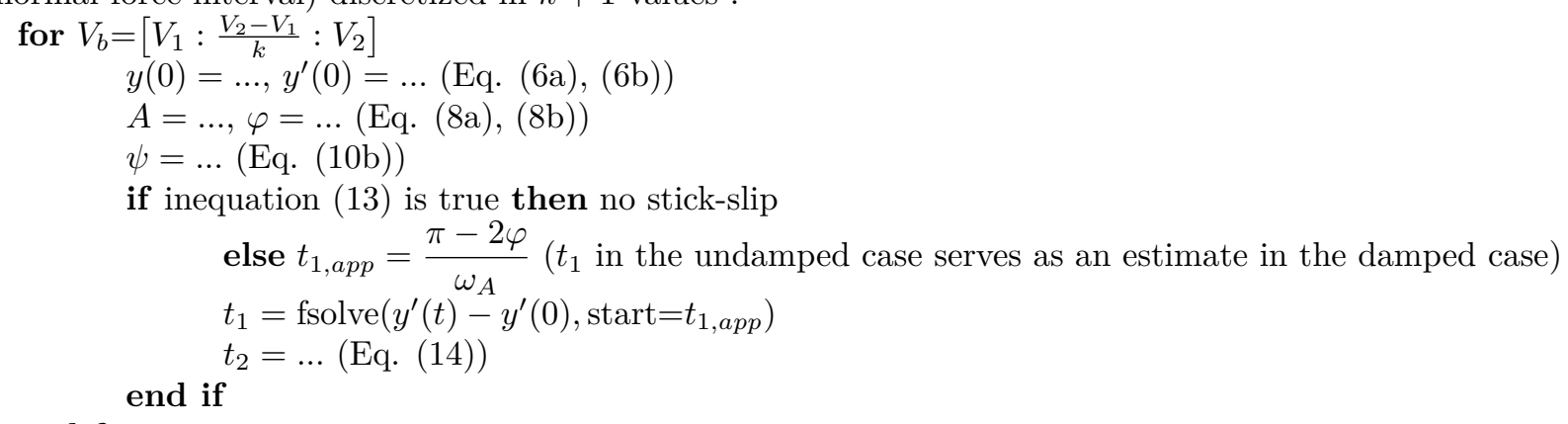

\section{end for}

The bifurcation diagram for this non-smooth law may include some non-smooth bifurcations that are not standard to smooth dynamical systems. This goes beyond the scope of this paper, however the book [16] may interest the reader.

\section{Regularized friction law}

We now present the regularized friction law $\mu_{n}\left(V_{r}\right)$ used in this paper. It is based on an analytical mother function $g$ and a regularization parameter $n$. This parameter $n$ acts as a scaling factor that modifies the stiffness of the regularization : large values of $n$ mean a highly nonlinear friction law. $g$ is defined as

$$
g\left(V_{r}\right):=\frac{-\mu_{d} V_{r} \sqrt{V_{r}^{2}+\varepsilon}-2 \alpha V_{r}}{V_{r}^{2}+1}, \quad \alpha=\sqrt{\mu_{s}\left(\mu_{s}-\mu_{d}\right)}
$$

where $\alpha$ and $\varepsilon$ are fixed parameters. The latter is meant to be small (here, $\varepsilon=10^{-4}$ ). The function $g$ is designed to verify the following properties :

- $g$ is odd ;

- $g\left(V_{r}\right) \rightarrow \mu_{d}$ when $V_{r} \rightarrow-\infty$;

- $\max g \rightarrow \mu_{s}$ when $\varepsilon \rightarrow 0$

Then, $\mu_{n}$ is defined as

$$
\mu_{n}\left(V_{r}\right):=g\left(n V_{r}\right)=\frac{-\mu_{d} V_{r} \sqrt{V_{r}^{2}+\frac{\varepsilon}{n^{2}}}-2 \frac{\alpha}{n} V_{r}}{V_{r}^{2}+\frac{1}{n^{2}}}
$$

A plot of Coulomb's law and the regularized law for several values of $n$, in Figure 3 , shows that $\mu_{n}\left(V_{r}\right)$ resembles Coulomb's law when $n$ is high. Additional details on the regularization process are given in AppendixA.

We highlight two main differences with Coulomb's friction law. The first one is a velocity-dependent dynamic coefficient. But as noted by Oden and Martins ([17], p. 548) the assumption of velocity independence is "now known to be invalid. A large volume of experimental data and empirical formulas for the variation of the friction coefficient with sliding velocity can be found". Yet Coulomb's law leads to more explicit expressions of the stick-slip solution, and for non-zero relative speed, it is the limit function of the sequence $\left(\mu_{n}\right)_{n>0}$.

The second difference is that $\mu_{n}$ is locally a decreasing function of $V_{r}$ around $V_{r}=0$, instead of a multivalued coefficient during adherence. This kind of smooth, velocity-dependent friction law, is a model commonly used to study the bowed string, from McIntyre and coll. [18] to recent sound synthesis [19]. The present study aims at investigating such a regularized law. More complex models have been designed ${ }^{2}$, to take into account effects due to rosin [21], string torsion [13], etc.

\footnotetext{
${ }^{2}$ Several bowed string models can be found in [20].
} 
a)

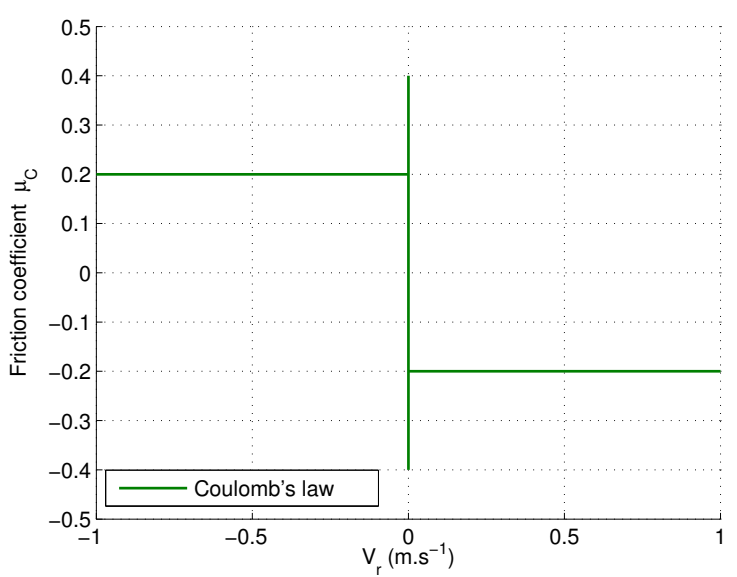

b)

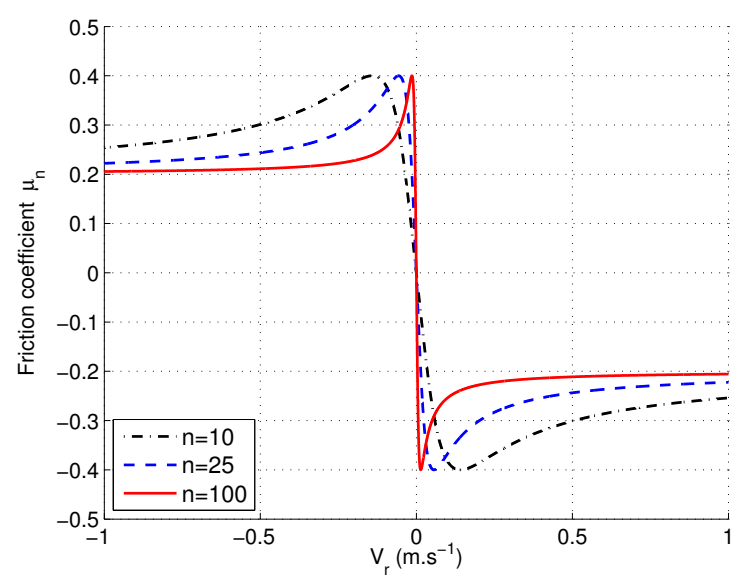

Figure 3: a) Coulomb's friction law, for $\mu_{s}=0.4, \mu_{d}=0.2$ b) Regularized friction $\mu_{n}$ for $n=10$ (black), 25 (blue), 100 (red).

A smooth friction law around 0 allows "no true sticking" (an expression found for example in [22]), a drawback in a purely static context, however the present paper focuses on dynamic phenomena. Friction regularization has been used to find approximate solutions of variational formulations (for example [23, 17]), but the authors found a small number of papers that use it in numerical simulations expecting realistic friction :

- Feeny and Moon [24] showed that for a harmonically forced spring-mass system, a smooth friction law can reproduce qualitatively the chaotic behaviour observed with Coulomb's law. The selected smooth function reads :

$$
\mu\left(V_{r}\right)=\left(\mu_{d}+\left(\mu_{s}-\mu_{d}\right) \operatorname{sech}\left(\beta V_{r}\right)\right) \tanh \left(\alpha V_{r}\right)
$$

- Quinn [25] proposes a smoothing procedure that respects the multivaluation at null relative speed by introducing an additional variable. However, it is presented in the case $\mu_{s}=\mu_{d}$, and its adaptation in the case $\mu_{s}>\mu_{d}$ may be delicate.

- Vrande et al. [26] use the function :

$$
\mu\left(V_{r}\right)=-\frac{2}{\pi} \frac{\arctan \left(\varepsilon V_{r}\right)}{1+\gamma\left|V_{r}\right|}
$$

where $\varepsilon$ is the regularization parameter. The maximal value of $\mu$ is not constant when $\varepsilon$ varies, and the range of belt speeds is restricted because this function tends to zero when $V_{r}$ tends to infinity.

\section{Periodic solutions using a regularized friction law}

We now investigate the periodic solutions with the regularized friction law presented in Section 4 . The numerical framework used is presented in [9] and [27] and implemented in the Matlab toolbox MANLAB [28].

\subsection{Quadratic recast}

Periodic solutions, and their continuation with respect to parameters $V_{b}$ or $F_{N}$, are studied with a continuation procedure. A robust method to compute a solution branch is the Asymptotic Numerical Method (ANM). It operates on first-order differential systems with quadratic nonlinearities. This is called a quadratic formulation and requires auxiliary variables to lower the derivation order to 1 , and to recast the nonlinearities into quadratic ones, so that $Z$, the vector of all variables, is solution of

$$
m\left(Z^{\prime}\right)=c_{0}+\lambda c_{1}+l_{0}(Z)+\lambda l_{1}(Z)+q(Z, Z)
$$


where $\lambda$ is the continuation parameter, $c_{0}$ and $c_{1}$ are vectors, $l_{0}, l_{1}$ and $m$ are linear operators, and $q$ is a bilinear operator [9]. After this quadratic recast, periodic solutions are studied using either the Harmonic Balance Method or the Orthogonal Collocation at Gauss points, as implemented in [27] ; unless stated otherwise below, they provide the same results. A detailed study of their convergence should be carried out in a companion paper [29].

The differential equation (1) on $y$ is transformed into a first-order system on $(y, z)$ :

$$
\begin{aligned}
& y^{\prime}=\omega_{1} z \\
& z^{\prime}=-2 \zeta_{1} \omega_{1} z-\omega_{1} y+\frac{F_{N}}{\omega_{1} M_{1}} \mu_{n}\left(V_{r}\right) \text { where } V_{r}=\omega_{1} z-V_{b}
\end{aligned}
$$

Then, seeing the definition (16) of $\mu_{n}\left(V_{r}\right)$, we define three auxiliary variables to obtain quadratic nonlinearities :

$$
\begin{gathered}
R=\sqrt{V_{r}^{2}+\frac{\varepsilon}{n^{2}}} \\
S=V_{r}^{2}+\frac{1}{n^{2}} \\
\mu_{n}=\frac{-\mu_{d} V_{r} R-2 \frac{\alpha}{n} V_{r}}{S}
\end{gathered}
$$

The definition of $R$ is then recasted as

$$
R^{2}=V_{r}^{2}+\frac{\varepsilon}{n^{2}}
$$

The latter, (25), is not equivalent to the previous definition, (22), and the positiveness of $R$ has to be checked (this should be studied in a companion paper [29]).

\subsection{Continuation with respect to the belt speed}

a)

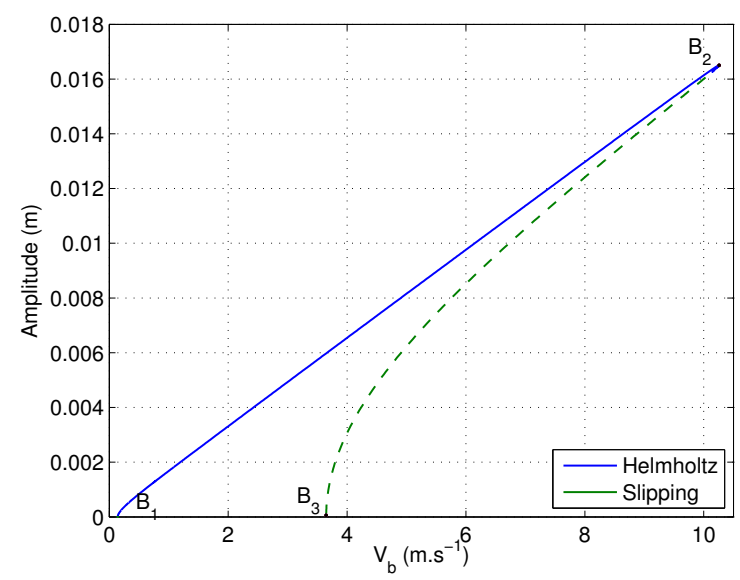

b)

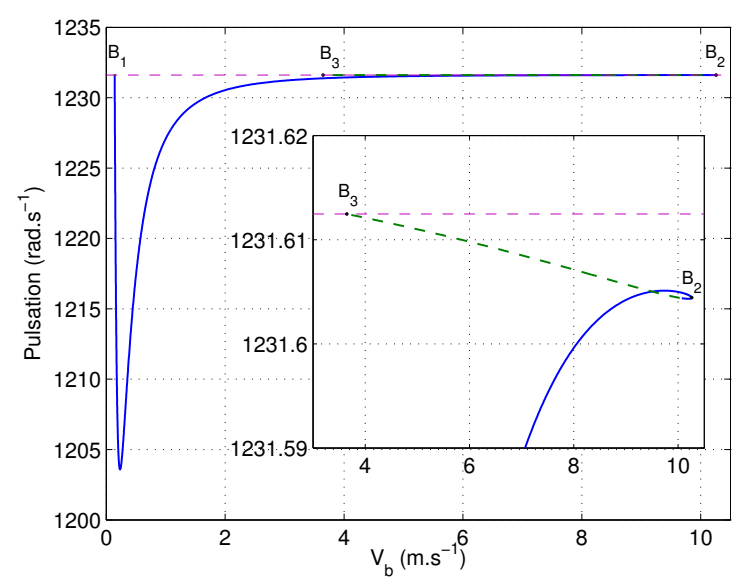

Figure 4: Bifurcation diagram with the continuation parameter $\lambda=V_{b}$, and $n=10, F_{N}=5 \mathrm{~N}$. The branch is plotted in solid line if it is stable, and dashed otherwise ; Helmholtz motion is in blue, slipping in green. HBM truncation order : $H=70$. a) Peak-to-peak amplitude versus the belt velocity. b) Pulsation versus the belt velocity. Natural pulsation $\omega_{1}$ is plotted in purple. A zoom window shows the branch between bifurcations $B_{2}$ and $B_{3}$.

A first continuation study is carried out with the belt speed $V_{b}$ being the continuation parameter $\lambda$, while the normal force is fixed $\left(F_{N}=5 \mathrm{~N}\right)$. The quadratic recast, emphasizing the operators of Eq. (19), and based on Eq. (20), (21), (23), (24) and (25), is : 


$$
\begin{array}{clll}
0=-\lambda & +\omega_{1} z-V_{r} & +0 \\
0=\frac{\varepsilon}{n^{2}} & +0 & +V_{r}^{2}-R^{2} \\
0 & =\frac{1}{n^{2}} & -S & +V_{r}^{2} \\
0 & =0 & +2 \frac{\alpha}{n} V_{r} & +\mu_{n} S+\mu_{d} V_{r} R \\
y^{\prime}=0 & +\underbrace{\omega_{1} z}_{m\left(Z^{\prime}\right)} & +0 \\
\underbrace{z^{\prime}}_{c_{0}+\lambda c_{1}} & -\underbrace{2 \zeta_{1} \omega_{1} z-\omega_{1} y+\frac{F_{N}}{\omega_{1} M_{1}} \mu_{n}}_{l_{0}(Z)+\lambda l_{1}(Z)} & +\underbrace{0}_{q(Z, Z)}
\end{array}
$$

For $n=10$, the bifurcation diagram is given in Figure 4 (left) with stability analysis and movement description. Amplitude refers to peak-to-peak amplitude of the displacement $y$.

The upper stable branch starts with a supercritical Hopf bifurcation ( $B_{1}$ on the diagram) around $V_{b}=0.14$ m.s ${ }^{-1}$, reaching a maximal belt speed $\left(B_{2}\right)$ around $V_{b}=10.25 \mathrm{~m} . \mathrm{s}^{-1}$. From there, the lower branch is unstable, and ends on a subcritical Hopf bifurcation $\left(B_{3}\right)$ around $V_{b}=3.65 \mathrm{~m} . \mathrm{s}^{-1}$. This stability analysis is performed with Hill's method [30]. This method is efficiently combined with the Harmonic Balance Method, provided that the Jacobian matrix of the nonlinear differential system $(y, z)^{\prime}=\mathcal{F}(y, z)$ (i.e. Eq. (20), (21)) is also quadratically recasted. In our case, this requires three additional variables, which means that the computation of the bifurcation diagram is slightly slowed down, then the stability analysis is almost immediate. This is a good compromise, and is preferred to the integration of the Jacobian matrix over one period to obtain the monodromy matrix, which is much more time consuming.

Some authors consider that using a regularized law prevents any real stick phase (for example, [22, 31]). We refine this by defining the movement type as follows : let $V_{n}$ be such that $\mu_{n}\left(V_{n}\right)=\max _{v} \mu_{n}(v)$, then

$$
\left|V_{r}(t)\right| \leqslant\left|V_{n}\right| \Rightarrow \text { stick, } \quad\left|V_{r}(t)\right|>\left|V_{n}\right| \Rightarrow \text { slip }
$$

Then, a solution is called either "slipping" if a period contains no sticking interval, or "Helmholtz motion", if there is exactly one sticking interval and one slipping interval. The plot in Figure (4) can be summed up as : Helmholtz motion on the upper branch, slipping on the lower branch. There are two details : around bifurcation $B_{1}$, the mass is only slipping ; the Helmholtz motion is still present on the lower branch near $B_{2}$.

We now choose greater values of $n$, the regularization parameter, namely 25, 50 and 100. The bifurcation diagram is altered (Figure 5):

- Hopf bifurcations $B_{1}$ and $B_{3}$ happen at lower belt speeds ;

- the maximal belt speed decreases ;

- the amplitude gap between the stable and the unstable branches decreases.

For belt speeds close to the first Hopf bifurcation, the motion amplitude is small and relative speed $V_{r}$ oscillates very slightly around $V_{n}$. In other words, $\mu_{n}$ stays close to its maximum $\mu_{s}$. In Coulomb's case, the amplitude of the stick-slip solution tends to a strictly positive value when the belt speed tends to 0 . There is a discontinuity at $V_{b}=0$, where the amplitude is zero since there is no motion. Examples of Helmholtz motion, in the regularized case and in Coulomb's case, are shown in Figure 6, a.

For Coulomb's law the pulsation is an increasing function of the belt speed (Figure 8) that tends to 0 when the belt speed tends to 0 . For the regularized law, pulsation starts at the natural pulsation at $B_{1}$, then decreases to similar values to the unregularized case, in a steep way for great values of $n$. From then on, it is also a function of $V_{b}$ increasing towards its final value $\omega_{1}$, reached at the second Hopf bifurcation $B_{3}$.

With greater values of $n$ (highly nonlinear system), for instance for $n=150$, the pulsation steep decreases near the first bifurcation $B_{1}$ ranges over a short interval, while the amplitude is a steep increasing function of the belt speed. In this interval, it is difficult for numerical methods to find the expected periodic solution. The Harmonic Balance Method predicts an hysteresis over a very short interval ; the size of this interval 


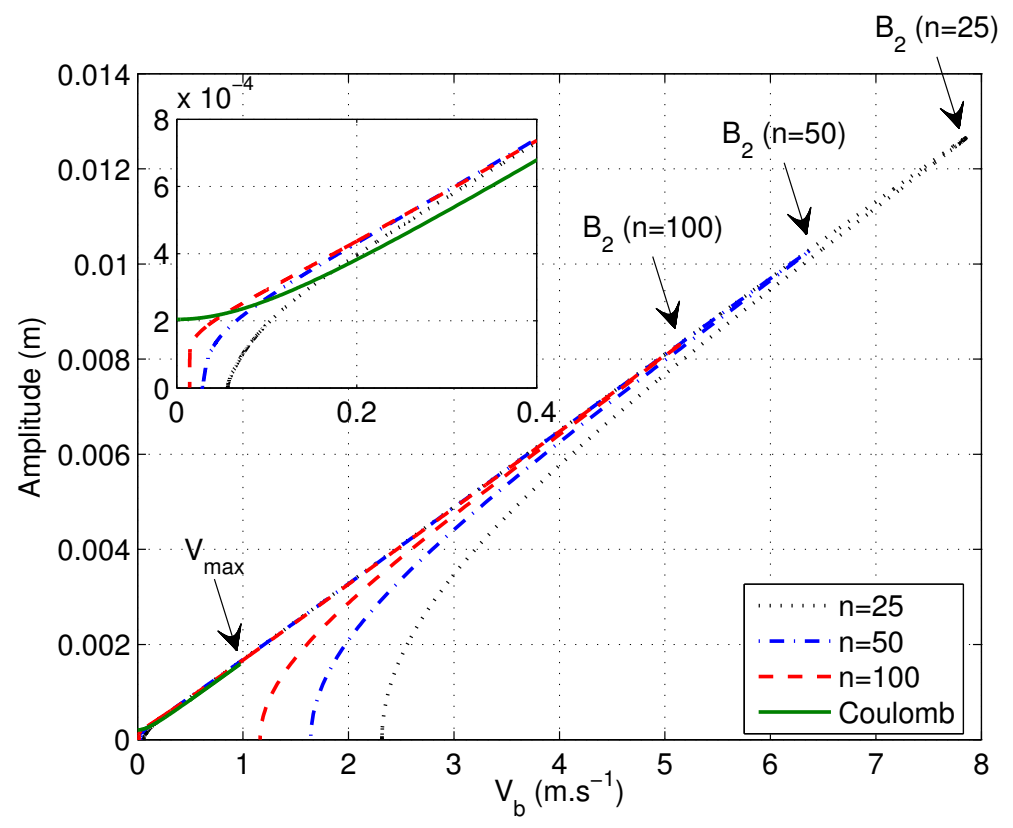

Figure 5: Bifurcation diagram (axes : belt speed, peak-to-peak amplitude of displacement) with the regularized friction law and $n=25$ (black; $H=100), n=50$ (blue; $H=125), n=100$ (red; $H=175$ ) ; with Coulomb's law (green). The position of the bifurcation points $B_{1}, B_{2}$ and $B_{3}$ change for each value of $n$. The maximal belt speed, examined in Section 3.3, is shown as $V_{\text {max }}$

a)

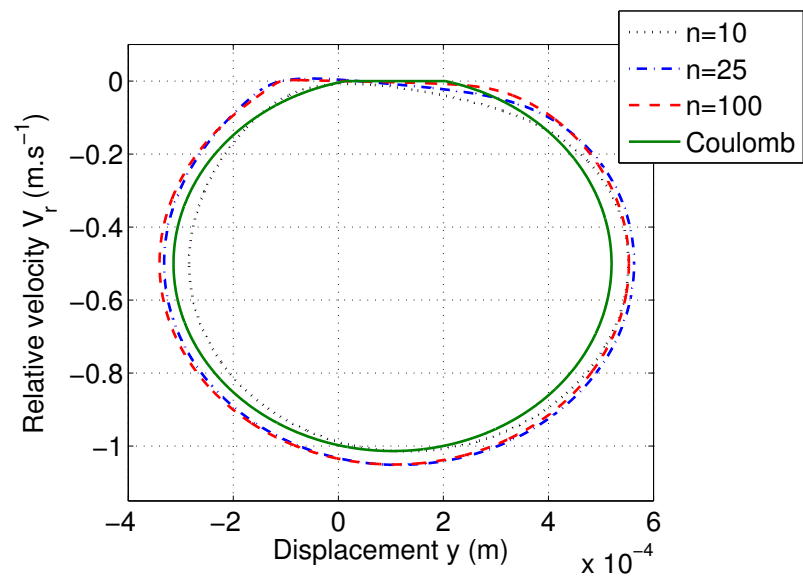

b)

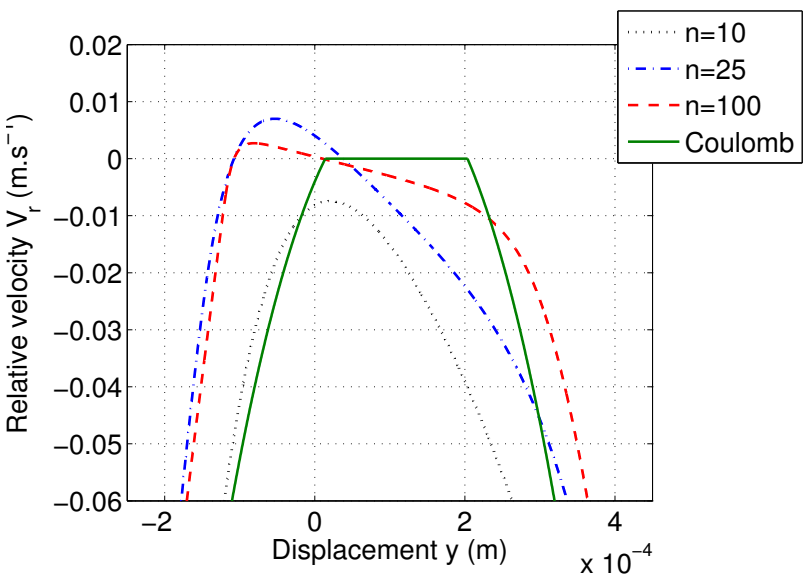

Figure 6: Phase diagrams, examples of stick-slip, for $V_{b}=0.5 \mathrm{~m} . \mathrm{s}^{-1}$. Regularized law : $n=10, H=70$, black; $n=25, H=100$, blue; $n=100, H=175$, red. Coulomb's law : green. a) Entire phase diagram. b) Zoom around the sticking part.

diminishes when more harmonics are used (Figure 9). The Orthogonal Collocation at Gauss points [32] does not exhibit this behaviour and describes amplitude and pulsation as functions of the continuation parameter $V_{b}$.

To confirm the continuation results, we can extract a periodic solution (from one of the two bifurcation diagrams, HBM or Collocation), and start a numerical solver with initial conditions $y(0), y^{\prime}(0)$. Among the solvers available in Matlab ODE suite [33] designed for stiff systems, ode15s was found to be reliable and faster than others on this system. Such a validation is presented for $n=100$ in Fig. 7 . However, for $n=150$ and in the $V_{b}$ interval described above, the absolute error tolerance of the function ode15s (parameter AbsTol) must be set as low as $10^{-14}$ (this worsens as $n$ increases since the system becomes stiffer). Otherwise, it does 


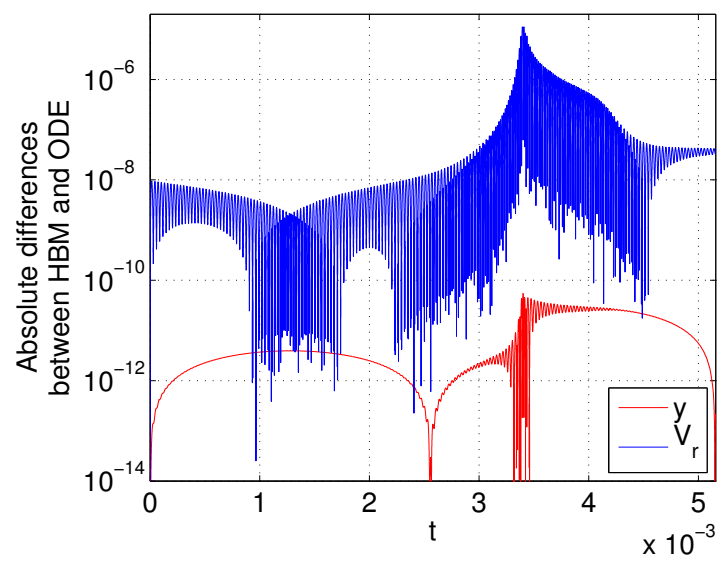

Figure 7: Validation with ode15s of the HBM solution, for $n=100$ and $H=175$, for $V_{b}=0.5$ m.s ${ }^{-1}$ : absolute differences between the two solutions, for $y$ and $V_{r}$.

not converge to the same periodic solution.

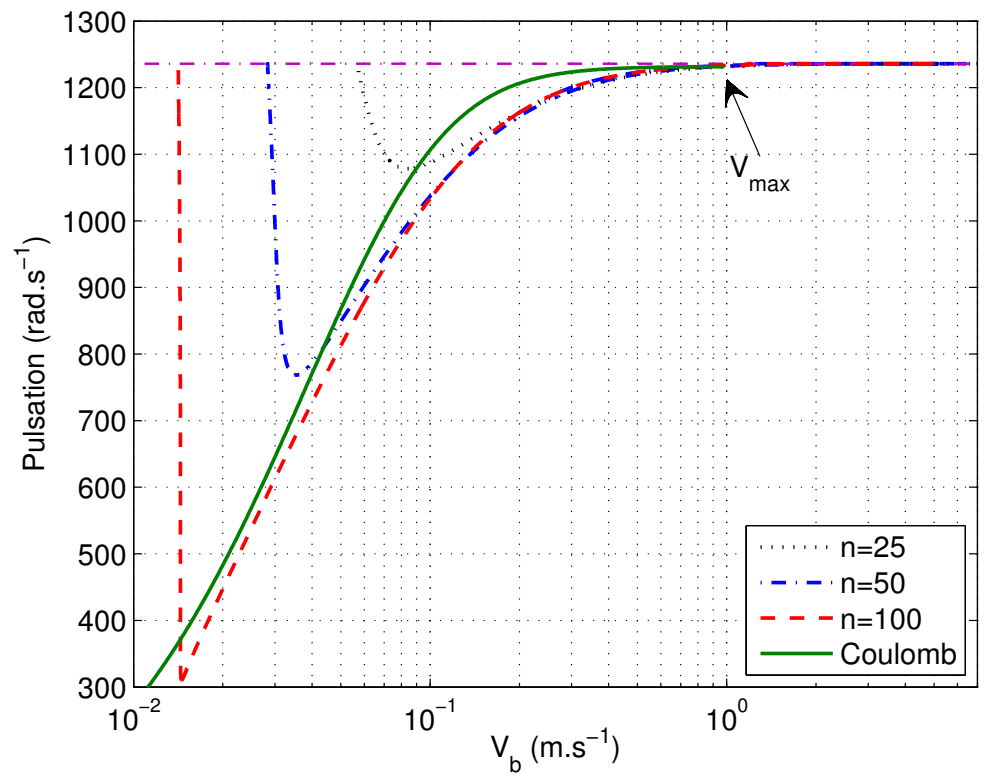

Figure 8: Pulsation versus belt speed, with the regularized friction law and $n=25$ (black; $H=100$ ), $n=50$ (blue; $H=125$ ), $n=100$ (red; $H=175)$; with Coulomb's law (green). Natural pulsation $\omega_{1}$ is drawn in purple. The branch between $B_{2}$ and $B_{3}$ is not visible at this scale.

\subsection{Continuation with respect to the normal force}

We now choose the continuation parameter $\lambda=F_{N}$ and a fixed belt speed $\left(V_{b}=0.2 \mathrm{~m} . \mathrm{s}^{-1}\right)$. Changing $\lambda$ requires a new quadratic formulation similar to Eqs. (26). It is not given here for sake of brevity.

For small values of $n$, for example $n=10$, the bifurcation diagram (Figure 10, a) shows a stable branch with a supercritical Hopf bifurcation. For higher values of $n(n=25$ is enough), the Hopf bifurcation becomes subcritical (Fig. 10, b). 
a)

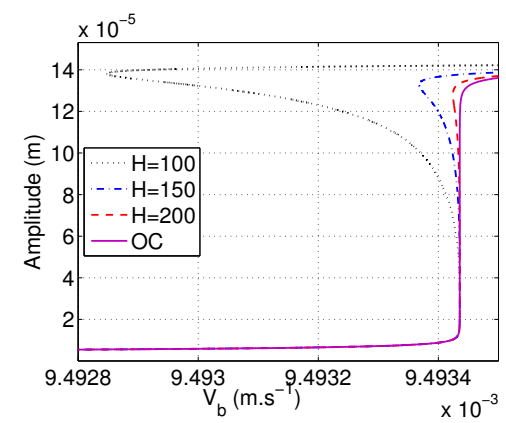

b)

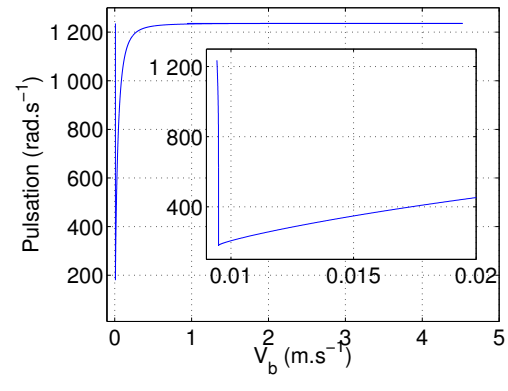

c)

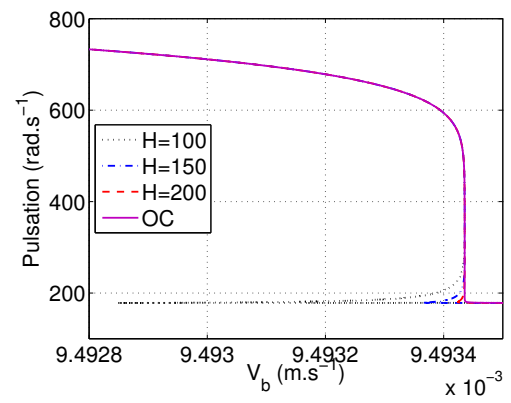

Figure 9: For $n=150$, comparison of the Harmonic Balance Method with $H=100$ (black), 150 (blue), 200 harmonics (red), and Orthogonal Collocation (OC) with $N_{\text {int }}=151$ subintervals. a) Peak-to-peak amplitude versus the belt velocity, for the interval $I=\left[9.4928 \times 10^{-3} ; 9.4935 \times 10^{-3}\right]$. b) Pulsation versus the belt velocity, entire diagram with a zoom window on the interval $[0.009 ; 0.02]$. c) Pulsation versus the belt velocity, zoom of the previous one on the interval $I=\left[9.4928 \times 10^{-3} ; 9.4935 \times 10^{-3}\right]$, comparison of the HBM and the OC.

Comparisons with Coulomb's law, for the amplitude (Figure 11) and the pulsation (fig. 12) show a qualitative agreement between regularized law and Coulomb's law. For either law, the mass can be carried away as far as desired during the stick phase, provided that the normal force $F_{N}$ is great enough. This phase becomes longer when $F_{N}$ increases, and therefore the pulsation decreases. Nevertheless, the agreement is less compelling than for the belt speed continuation. For small values of the regularization parameter $n$, the amplitude is close to the amplitude occurring with Coulomb's law. For $n=50$ or $n=100$ and high values of normal force $F_{N}$, there is still a large difference between pulsation with Coulomb's law and the one with regularized law.

a)

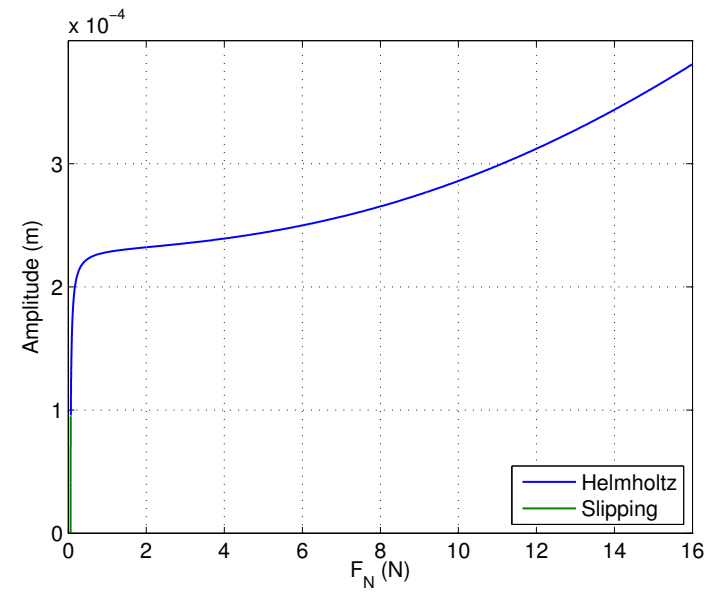

b)

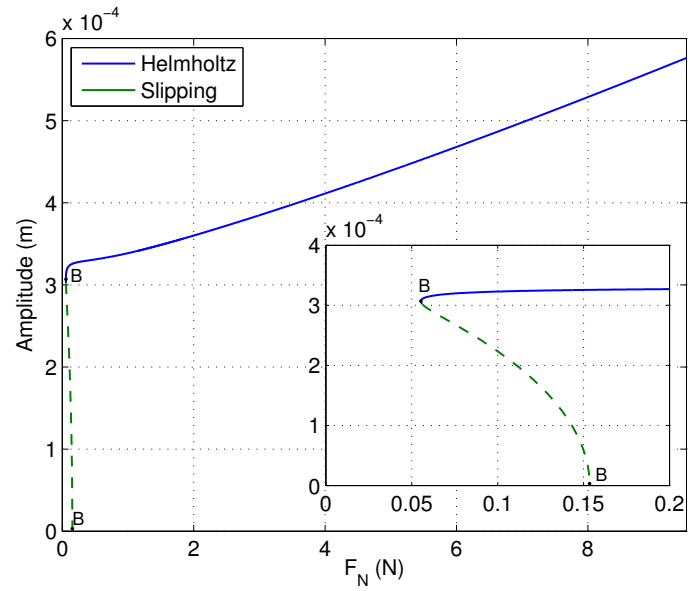

Figure 10: Bifurcation diagram with the continuation parameter $\lambda=F_{N}$. The branch is plotted in solid line if it is stable, and dashed otherwise ; Helmholtz motion is in blue, slipping in green. a) Weakly nonlinear system, $n=10$. HBM truncation order : $H=70$. b) Stiff nonlinear system, $n=100 . H=300$.

\section{Conclusion}

In this paper we investigated the periodic solutions of a mass-spring-damper-belt system, and compared two different friction laws, Coulomb's law and a regularized law. In the Coulomb case, the stick-slip solution is constructed sequentially, giving access to limit values for the belt speed and the normal force. In the regularized case, the bifurcation diagrams, obtained by numerical continuation with respect to the belt speed 


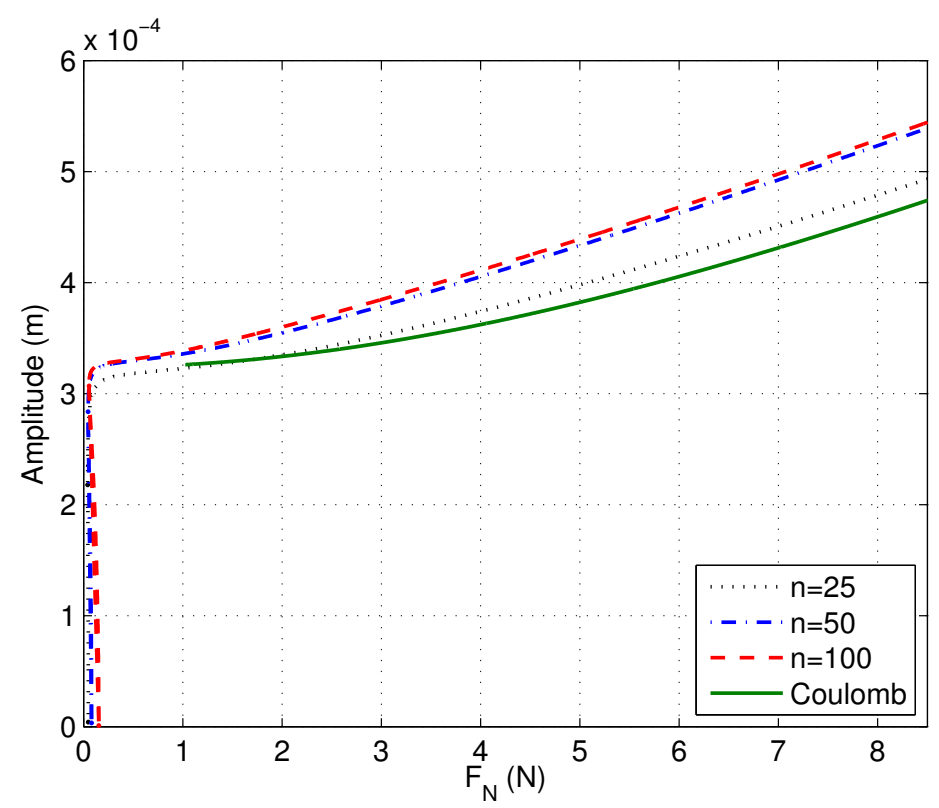

Figure 11: Peak-to-peak amplitude versus normal force, with the regularized friction law and $n=25$ (black, $H=70$ ), $n=50$ (blue, $H=150$ ), $n=100$ (red, $H=300$ ) ; with Coulomb's law (green).

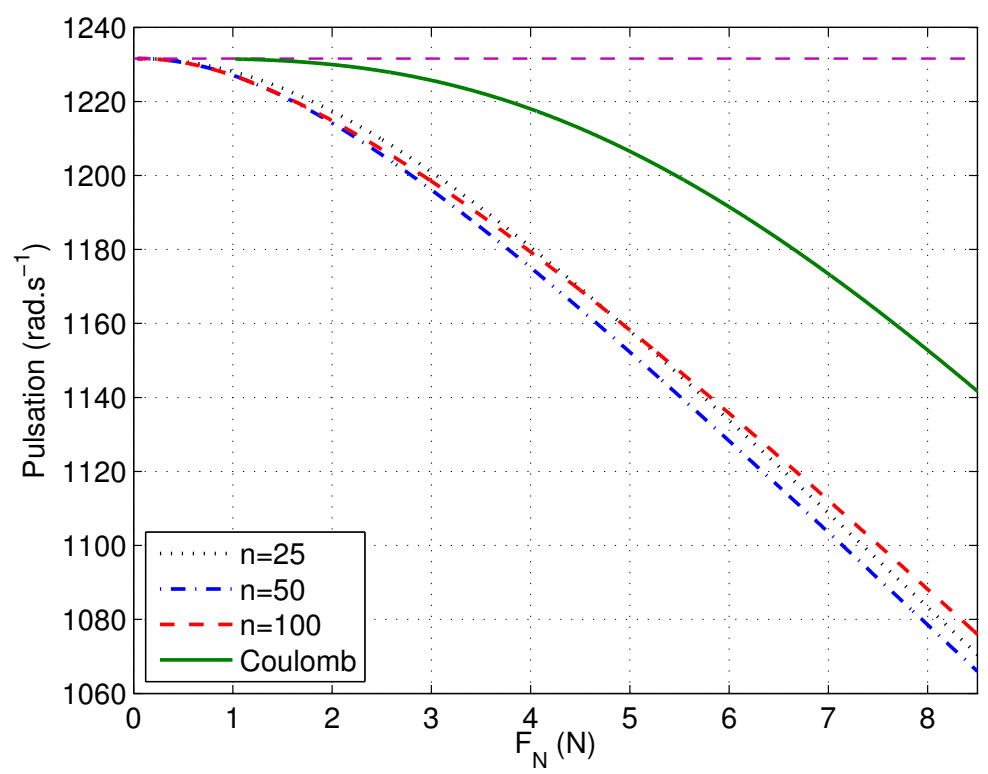

Figure 12: Pulsation versus normal force, with the regularized friction law and $n=25$ (black, $H=70$ ), $n=50$ (blue, $H=150$ ), $n=100$ (red, $H=300$ ) ; with Coulomb's law (green). Natural pulsation $\omega_{1}$ is shown in purple.

or to the normal force, and for several values of the regularization parameter, fulfilled two goals. They prove the robustness of the association of the Asymptotic Numerical Method and the HBM confronted with a highly nonlinear law; they give a comprehensive description of the stick-slip branch and of the slipping branch, as well as their evolution when the system becomes highly nonlinear. Formulations from the Coulomb case give the means of a comparison between the regularized solutions and a standard reference. 
The present study highlights that several qualitative aspects of friction are preserved with the smooth function of relative velocity chosen as the friction law. With an appropriate definition, stick-slip motion exists, its amplitude increases with respect to the belt speed and its pulsation decreases with respect to the normal force. There are limit values for the belt speed and the normal force beyond which this periodic motion ceases to exist. However, with the regularized law some unstable branches of solution can exist, and have no counterpart with Coulomb's law. For example, the continuation with respect to the belt speed shows a branch of slipping solution.

Thanks to its important nonlinearity, this regularized system serves as a benchmark for our methods of time discretization. A future companion paper [29] will compare the Harmonic Balance Method and the Orthogonal Collocation at Gauss points, for several values of the regularization parameter $n$. Future works based on this regularized system can feature more complete models, either for the string or the friction law.

\section{Acknowledgements}

This work has been carried out in the framework of the Labex MEC (ANR-10-LABX-0092) and of the A*MIDEX project (ANR-11-IDEX-0001-02), funded by the Investissements d'Avenir French Government program managed by the French National Research Agency (ANR).

\section{AppendixA. Regularization construction}

The regularized law $\mu$ can be designed as follows : $\mu$ is an odd, analytical function, with a (single) maximum value $\mu_{s}$ on $\mathbb{R}_{-}$, and its asymptotic value is $\mu_{d}$ (when $V_{r} \rightarrow-\infty$ ). The definition

$$
\forall V_{r} \leqslant 0, \quad \mu\left(V_{r}\right)=\frac{-\mu_{d} V_{r}^{2}-2 \alpha V_{r}}{V_{r}^{2}+1},
$$

verifies our constraints, provided that $\alpha=\sqrt{\mu_{s}\left(\mu_{s}-\mu_{d}\right)}$. Since $V_{r} \leqslant 0$ with Coulomb's law, it may seem sufficient to define $\mu$ with Eq. (A.1). Yet, using this definition, numerical simulation shows that $V_{r}$ does not stay negative over one period, for large intervals of belt speed and normal force. Since the expression given in Eq. (A.1) is not odd, the friction coefficient is incorrect over a fraction of the period. A satisfying replacement would be, for all $V_{r}$

$$
\mu\left(V_{r}\right)=\frac{-\mu_{d} V_{r}\left|V_{r}\right|-2 \alpha V_{r}}{V_{r}^{2}+1}
$$

Unfortunately the modulus $\left|V_{r}\right|$ in Eq. (A.2) is not smooth enough for the continuation study, so we choose $\sqrt{V_{r}^{2}+\varepsilon}$ instead. The constant $\alpha$ gives the correct maximum $\mu_{s}$ only for $\varepsilon=0$, and small values of $\varepsilon$ engender slightly exaggerated values for the maximum. Numerically, the difference between the smooth function $g$, defined in Eq. (15), and the function $\mu$ defined in Eq.(A.2), is one order of magnitude smaller than $\varepsilon$. Thus, the chosen value $\varepsilon=10^{-4}$ in this paper gives $g$ a maximum value of $0.4+10^{-5}$, close to $\mu_{s}=0.4$, as shown in figure A.13 by computing the difference between $g$ and $\mu$.

\section{AppendixB. Jacobian matrix}

The first-order differential system (Eqs. (20), (21)) reads

$$
\left(\begin{array}{l}
y \\
z
\end{array}\right)^{\prime}=\left(\begin{array}{c}
\mathcal{F}_{1}(y, z) \\
\mathcal{F}_{2}(y, z)
\end{array}\right):=\left(\begin{array}{c}
\omega_{1} z \\
-2 \zeta_{1} \omega_{1} z-\omega_{1} y+\frac{F_{N}}{\omega_{1} M_{1}} \mu_{n}
\end{array}\right)
$$

The partial derivatives of $\mathcal{F}_{1}$ and $\mathcal{F}_{2}$ are

$$
\begin{aligned}
& \frac{\partial \mathcal{F}_{1}}{\partial y}=0, \quad \frac{\partial \mathcal{F}_{1}}{\partial z}=\omega_{1} \\
& \frac{\partial \mathcal{F}_{2}}{\partial y}=-\omega_{1}, \quad \frac{\partial \mathcal{F}_{2}}{\partial z}=-2 \zeta_{1} \omega_{1}+\frac{F_{N}}{\omega_{1} M_{1}} \frac{\mathrm{d} V_{r}}{\mathrm{~d} z} \frac{\mathrm{d} \mu_{n}\left(V_{r}\right)}{\mathrm{d} V_{r}} \quad \text { (chain rule) }
\end{aligned}
$$




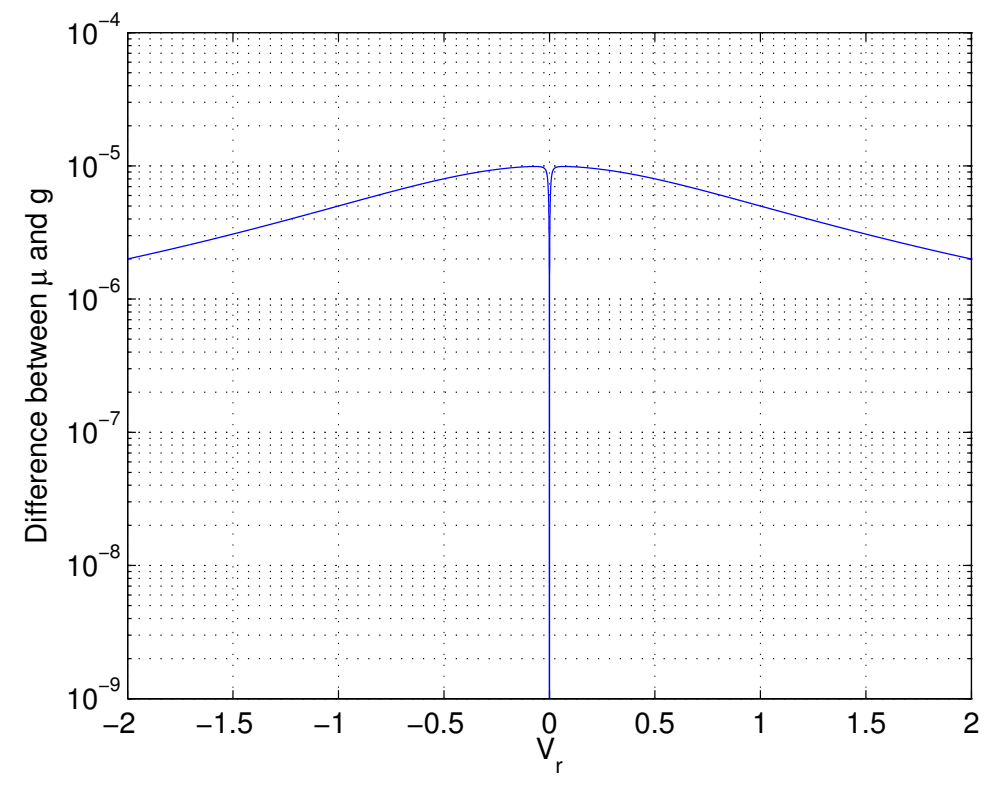

Figure A.13: Absolute difference between the smooth function $g$, defined in Eq. (15), and the function $\mu$ defined in Eq.(A.2), for $\varepsilon=10^{-4}$.

where

$$
\frac{\mathrm{d} V_{r}}{\mathrm{~d} z}=\omega_{1}, \quad \frac{\mathrm{d} \mu_{n}}{V_{r}}=\frac{1}{S}\left(-\mu_{d} R-\mu_{d} \frac{V_{r}^{2}}{R}-2 \frac{\alpha}{n}-2 V_{r} \mu_{n}\right)
$$

Three auxiliary variables are defined:

$$
U_{R}=\frac{V_{r}^{2}}{R}, \quad U_{S}=\frac{1}{S}, \quad U_{M}=V_{r} \mu_{n}
$$

The Jacobian matrix of system (B.1) can then be written with the following constant, linear and quadratical matrices:

$$
\left(\begin{array}{cc}
0 & \omega_{1} \\
-\omega_{1} & -2 \zeta_{1} \omega_{1}
\end{array}\right)+\left(\begin{array}{cc}
0 & 0 \\
0 & -2 \frac{F_{N} \alpha}{M_{1} n} U_{S}
\end{array}\right)+\frac{F_{N}}{M_{1}}\left(\begin{array}{cc}
0 & 0 \\
0 & -\mu_{d} U_{S} R-\mu_{d} U_{S} U_{R}-2 U_{S} U_{M}
\end{array}\right)
$$

\section{References}

[1] B. Feeny, A. Guran, N. Hinrichs, K. Popp. A historical review on dry friction and stick-slip phenomena, ASME Applied Mechanics Review 51 (5), p. 321-341, 1998.

[2] A. Papinniemi, J.C.S. Lai, J. Zhao, L. Loader. Brake squeal: a literature review, Applied Acoustics 63, p. 391-400, 2002.

[3] L. Gaul, R. Nitsche. The role of friction in mechanical joints. Applied Mechanics Review, 54 (2), p. 93-106, 2001.

[4] A. Akay. Acoustics of friction, Jounal of the Acoustical Society of America, 111 (4), p. 1525-1548, 2002.

[5] E. Schoonderwaldt, K. Guettler, A. Askenfelt. An empirical investigation of bow-force limits in the Schelleng diagram, Acta Acustica united with Acustica, 94, p. 604-622, 2008. 
[6] J. Woodhouse, P.M. Galluzzo. The bowed string as we know it today, Acta Acustica united with Acustica 90, p. 579-589, 2004.

[7] O. Inácio, J. Antunes. A linearized modal analysis of the bowed string, Proceedings of the International Congress of Acoustics. SEA, Madrid. 2007. URL <http://www.seaacustica.es/WEB_ICA_07/fchrs/papers/mus-04-002.pdf> (accessed 17 March 2016)

[8] P. Vigué, B. Cochelin, S. Karkar, C. Vergez. Investigation of periodic solutions of a bowed string toy model, in M02 Mini symposium Mécanique des Instruments de Musique, Association Française de Mécanique, 2015. URL < http://hdl.handle.net/2042/56983> (accessed 17 March 2016)

[9] B. Cochelin, C. Vergez. A high order purely frequency-based harmonic balance formulation for continuation of periodic solutions, Journal of Sound and Vibration, 324, p. 243-262, 2009.

[10] S. Karkar, B. Cochelin, C. Vergez. A high-order, purely frequency based harmonic balance formulation for continuation of periodic solutions: The case of non-polynomial nonlinearities, Journal of Sound and Vibration, 332, p.968-977, 2013.

[11] E. Pennestri, V. Rossi, P. Salvini, P. P. Valentini. Review and comparison of dry friction force models, Nonlinear Dynamics, p. 1-17, 2015.

[12] D. Karnopp. Computer simulation of stick-slip friction in mechanical dynamic systems, Journal of Dynamic Systems, Measurement and Control, 107 (1), p. 100-103, 1985.

[13] R. I. Leine, D. H. Van Campen, A. De Kraker, L. Van Den Steen. Stick-slip vibrations induced by alternate friction models, Nonlinear Dynamics 16, p. 41-54, 1998.

[14] U. Andreaus, P. Casini. Dynamics of friction oscillators excited by a moving base and/or driving force, Journal of Sound and Vibration, 245 (4), p. 685-699, 2001.

[15] C. Wensrich. Slip-stick motion in harmonic oscillator chains subject to Coulomb friction, Tribology International, 39, p. 490-495, 2006.

[16] R. I. Leine, H. Nijmeijer. Dynamics and bifurcations of non-smooth mechanical systems, Lecture Notes in Applied and Computational Mechanics, vol. 18, Springer, 2004.

[17] J. T. Oden, J. A. C. Martins. Models and computational methods for dynamic friction phenomena, Computer methods in applied mechanics and engineering 52, p. 527-634, 1985.

[18] M. E. McIntyre, R. T. Schumacher, J. Woodhouse. On the oscillations of musical instruments, Journal of the Acoustical Society of America, 74, p. 1325-1345, 1983.

[19] C. Desvages, S. Bilbao. Physical modeling of nonlinear player-string interactions in bowed string sound synthesis using finite difference methods, Proceedings ISMA, p. 261-266, 2014.

[20] J. Woodhouse. Idealised models of a bowed string, Acustica 79, p. 233-250, 1993.

[21] J. H. Smith, J. Woodhouse. The tribology of rosin, Journal of the Mechanics and Physics of Solids, 48, p. 1633-1681, 2000.

[22] E. J. Berger. Friction modeling for dynamic system simulation, Applied Mechanics Review, 55, 6, p. 535-577, 2002.

[23] J. A. C. Martins, J. T. Oden. A numerical analysis of a class of problems in elastodynamics with friction, Computer Methods in Applied Mechanics and Engineering, 40, p. 327-360, 1983.

[24] B. Feeny, F. C. Moon. Chaos in a forced dry-friction oscillator : experiments and numerical modelling, Journal of Sound and Vibration, 170 (3), p. 303-323, 1994.

[25] D. D. Quinn. A new regularization of Coulomb friction, Journal of Vibration and Acoustics, 126, p. 391-397, 2004. 
[26] B. L. Van De Vrande, D. H. Van Campen, A. De Karker. An approximate analysis of dry-friction-induced stick-slip vibrations by a smoothing procedure, Nonlinear Dynamics, 19, p. 157-169, 1999.

[27] S. Karkar, C. Vergez, B. Cochelin. A comparative study of the harmonic balance method and the orthogonal collocation method on stiff nonlinear systems, Journal of Sound and Vibration, 333, 12, p.2554-2567, 2014.

[28] S. Karkar, R. Arquier, B. Cochelin, C. Vergez, A. Lazarus, O. Thomas, MANLAB, An Interactive Continuation Software. URL <http://manlab.lma.cnrs-mrs.fr> (accessed 17 March 2016)

[29] P. Vigué, C. Vergez, S. Karkar, B. Cochelin. Regularized friction and continuation : A comparative study of the harmonic balance method and the orthogonal collocation method, to be submitted.

[30] A. Lazarus, O. Thomas. A harmonic-based method for computing the stability of periodic solutions of dynamical systems, Comptes Rendus Mecanique 338, p. 510-517, 2010.

[31] K. Popp, N. Hinrichs, M. Oestreich. Analysis of a self excited friction oscillator, Dynamics with Friction: Modeling, Analysis and Experiments, p. 1-35, World Scientific Publishing Company.

[32] C. de Boor, B. Swartz. Collocation at Gaussian points, SIAM Journal of Numerical Analysis, 10, p. 582-606, 1973.

[33] L.F. Shampine, M.W. Reichelt. The Matlab ODE suite, SIAM Journal on Scientific Computing, 18, p. 1-22, 1997. 